GU J Sci, Part C, 6(3): 680-690 (2018)

Gazi Üniversitesi
Fen Bilimleri Dergisi
PART C: TASARIM VE TEKNOLOJI
dergipark.gov.tr/http-gujsc-gazi-edu-tr

\title{
Al/PVA (Zn-katkılı)/p-Si (MPS) Yapılarda Organik Arayüzey Tabaka Kalınlığının ve Seri Direncin C-G/ $\omega$-V Karakteristikleri Üzerine Etkisi
}

\author{
Hüseyin TECIMER ${ }^{1, *}$ \\ ${ }^{I}$ Karabük Üniversitesi, Teknoloji Fakültesi, Mekatronik Mühendisliği Bölümü, 78050, Merkez/KARABÜK
}

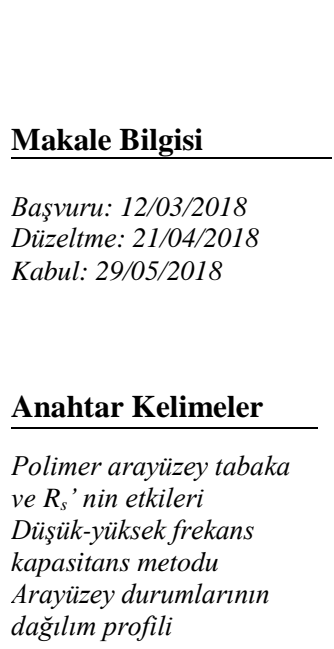

Keywords

The effects of interfacial layer and $R_{s}$

Low-high frequency capacitance method Distribution interfacial of interface states.

\begin{abstract}
$\ddot{O} \mathbf{z}$
Bu çalışmada, 50 ve $150 \mathrm{~nm}$ kalınlıklarına sahip (Zn-katkılı PVA) polimer ara yüzey tabakalı $\mathrm{Al} / \mathrm{p}-\mathrm{Si}$ (MPS) yapıların elektriksel özellikleri, kapasitans-kondüktans-voltaj (C-G/ $\omega-\mathrm{V})$ ölçüm metodu kullanılarak frekansa bağlı incelendi. C-G/ $\omega-\mathrm{V}$ ölçümleri $-3.5 /+5.5 \mathrm{~V}$ aralığında $50 \mathrm{mV}$ adımlarla $2 \mathrm{kHz}, 20 \mathrm{kHz}$ ve $200 \mathrm{kHz}$ frekanslarında oda sıcaklığında alındı. Ters öngerilim $\mathrm{C}^{-2}-\mathrm{V}$ grafiklerinin lineer kısmının kesim noktası ve eğimlerinden faydalanılarak; difüzyon potansiyeli $\left(\mathrm{V}_{\mathrm{D}}\right.$,) alıcı katkı atomlarının yoğunluğu $\left(\mathrm{N}_{\mathrm{A}}\right)$, Fermi enerji $\left(\mathrm{E}_{\mathrm{F}}\right)$ seviyesi, tüketim tabakasının kalınlığ $\left(\mathrm{W}_{\mathrm{D}}\right)$ ve potansiyel engel yüksekliği $\left(\Phi_{\mathrm{B}}\right)$ gibi temel elektriksel parametreler hem frekansa hem de kalınlığa bağlı olarak elde edilerek karşılaştırıldı. Bu parametrelerin tümü oldukça hem organik arayüzey tabakasının kalınlığına hem de frekansa bağlı değişiklik göstermektedir. Ayrıca Nicollian ve Brews metodu kullanılarak bu yapıların direnci $\left(\mathrm{R}_{\mathrm{i}}\right)$ ve düşük-yüksek frekans $\left(\mathrm{C}_{\mathrm{LF}}-\mathrm{C}_{\mathrm{HF}}\right)$ kapasitans metodu kullanılarak da arayüzey durumlarının $\left(\mathrm{N}_{\mathrm{ss}}\right)$ voltaja bağlı değişim profilleri elde edildi. Seri direnç $\left(\mathrm{R}_{\mathrm{s}}\right)$ etkisinin hangi bölge ve frekansta daha etkin olduğunu belirlemek için yapıların ölçülen $C-G / \omega-V$ eğrileri $R_{s}$ değeri dikkate alınarak düzeltildi. Elde edilen deneysel sonuçlar hem polimer arayüzey tabaka kalınlığının hem de $R_{s}$ ve $N_{s s}$ değerlerinin $C-G / \omega-V$ ölçümleri üzerinde oldukça etkili olduğu gözlendi.
\end{abstract}

\section{The Effect of Organic Polymer Interfacial Layer Thickness and Series Resistance C-G/ $\omega$-V Characteristics in Al/PVA (Zn- doped)/p-Si (MPS) Structure}

\begin{abstract}
In this study, the electrical properties of polymer interfacial layer Al/p-Si (MPS) with thickness of 50 and $150 \mathrm{~nm}$ ( $\mathrm{Zn}$-doped PVA) were investigated in terms of frequency using the capacitance- conductance-voltage $(\mathrm{C}-\mathrm{G} / \omega-\mathrm{V})$ measurement methods. $\mathrm{C}-\mathrm{G} / \omega-\mathrm{V}$ measurements were taken at room temperature at frequencies of $2 \mathrm{kHz}, 20 \mathrm{kHz}$ and $200 \mathrm{kHz}$ in the range of -3.5 I + $5.5 \mathrm{~V}$ with $50 \mathrm{mV}$ steps. Utilizing the cut-off point and slope of the linear part of the reverse biased $\mathrm{C}^{-2}-\mathrm{V}$ graphics; basic electrical parameters such as the values of diffusion potential $\left(\mathrm{V}_{\mathrm{D}}\right)$, doping concentration of acceptor atoms $\left(\mathrm{N}_{\mathrm{A}}\right)$, Fermi energy level $\left(\mathrm{E}_{\mathrm{F}}\right)$, depletion layer width $\left(\mathrm{W}_{\mathrm{D}}\right)$ and barrier height $\left(\Phi_{\mathrm{B}}\right)$ were obtained and compared based on both frequency and thickness. These parameters are highly dependent on both the thickness of organic interface layer and frequency. In addition, voltage-dependent change profiles of interface states $\left(\mathrm{N}_{\mathrm{ss}}\right)$ and the resistance $\left(\mathrm{R}_{\mathrm{i}}\right)$ were obtained using low-high frequency $\left(\mathrm{C}_{\mathrm{LF}}-\mathrm{C}_{\mathrm{HF}}\right)$ capacitance method and the Nicollian and Brews method of these structures using, respectively. To determine which region and frequency of the series resistance $\left(R_{s}\right)$ effect is more effective, measured C-G/ $\omega-\mathrm{V}$ curves were corrected considering $\mathrm{R}_{\mathrm{s}}$ value. Obtained experimental results were observed to be very effective on the $C-G / \omega-V$ of the polymer interfacial layer thickness and $R_{s}$ and $N_{s s}$ values.
\end{abstract}

\section{GİRIŞ (INTRODUCTION)}

Son yıllarda, metal-polimer-yarıiletken (MPS) tipi yapılar üzerine deneysel ve teorik çalışmalar artmış olup bu yapıların yarıiletken teknolojisinde kullanımı oldukça yaygınlaşmış̧ır. [1-5]. Organik yarıiletkenler; düşük maliyetli, kolay üretim süreci, esnek olmaları gibi önemli özelliklerinden dolay1 yarıiletken teknolojisinde kullanılan inorganik yarıiletkenlere kıyasla oldukça avantajlara sahiptir. $\mathrm{Bu}$ 
nedenle organik 1şık yayan diyot (OLED), Schottky engel diyot (SBDs) gibi elektronik elemanların üretiminde çekici hale gelmiştir [5-8].

MPS yapılar hazırlanırken, yariiletkenin tipine uygun iş fonksiyonlu metaller seçilerek doğrultucu kontak oluşturulur. Yariiletken p-tipi olduğunda genellikle metal olarak yüksek saflıkta alüminyum (Al) seçilir. Yarıiletken malzeme olarak da kararlı ve ucuz olduğu için Si tercih edilir. Ayrıca, yapının performansını artırmak ve yük geçişlerini düzenlemek için, M/S arayüzeyine yüzeyi pasivize eden, sızıntı akımını düşüren ve doğrultma özelliğine sahip organik arayüzey tabaka büyütülür.

Organik arayüzey tabakasının kullanılmasının en büyük avantajı kaplama tekniklerinin kolay olmasından dolayı üretim masraflarını düşürmesidir [1-5, 9-10]. Endüstriyel uygulamalarda sıkça kullanılan zayıf iletkenliğe sahip Polyvinil alkol (PVA), farklı metallerle katkılanarak bu özelliği artırılabilir. Bahsedilen nedenlerden dolayı çalışmada, organik arayüzey tabakası olarak PVA ve polimerin iletkenliğini artırmak için Zn katkısı kullanıldı.

MPS yapıların elektriksel karakteristikleri, özellikle arayüzey polimer tabakanın oluşumuna, M/S arasındaki arayüzey durumlarının özelliklerine, $R_{s}$ ve Schottky engel yüksekliklerindeki homojensizliğe bağlıdır [1-3]. Bu nedenle Al/p-Si (MS) tipi yapıların performansını artırmak amacıyla iki farklı kalınlıkta (Zn-katkılı PVA) polimer arayüzey tabakası kullanıldı. Arayüzey tabakasının kalınlığı, MPS ${ }_{1}$ yapısı için $50 \mathrm{~nm}$ ve $\mathrm{MPS}_{2}$ yapısı için $150 \mathrm{~nm}$ 'dir. Hazırlanan bu MPS 1 ve $\mathrm{MPS}_{2}$ yapılarının temel elektriksel parametreleri, farklı frekans ve gerilim altında $\mathrm{C}-\mathrm{G} / \omega-\mathrm{V}$ ölçümleri kullanılarak kıyaslamalı olarak incelendi. $\mathrm{M} / \mathrm{S}$ arayüzeyindeki polimer tabakanın varlığı arayüzey durumlarını etkiler ve bu yapıların elektriksel parametrelerini önemli ölçüde değiştirir. Bu yüzden, Nicollian ve Brews metodu kullanarak bu yapıların direnci ve düşük-yüksek frekans $\left(\mathrm{C}_{\mathrm{LF}}-\mathrm{C}_{\mathrm{HF}}\right)$ kapasitans metodu kullanarak da voltaja bağlı arayüzey durumlarının değişim profili elde edildi. Ek olarak $R_{s}$ ' nin hangi bölge ve frekansta etkili olduğunu belirlemek amacıyla MPS yapıların ölçülen $\mathrm{C}-\mathrm{G} / \omega-\mathrm{V}$ eğrileri seri direnç değeri dikkate alınarak düzeltildi. C-G/ $\omega-\mathrm{V}$ ölçümlerinin organik arayüzey tabakasının kalınlığına, yapının direncine ve arayüzey dağılımlarına bağl1 olduğu görüldü.

\section{MATERYAL ve METOD (MATERIALS AND METHODS)}

Al/(Zn-katkılı PVA)/p-Si (MPS) yapısının hazırlanmasında alttaş olarak (100) yönelimli, $350 \mu \mathrm{m}$ kalınlıkl1, $5.08 \mathrm{~cm}$ çap ve $0.01 \Omega . \mathrm{cm}$ özdirence sahip bor-katkılı p-Si kullanıldı. p-Si alttaş RCA prosedürü ile temizlendikten sonra kuru azot gazı kullanılarak kurutuldu. Alttaş 60s süresince $\mathrm{H}_{2} \mathrm{SO}_{4}: \mathrm{H}_{2} \mathrm{O}_{2}: \mathrm{H}_{2} \mathrm{O}=3: 1: 1$ oranında asit çözeltisi ile aşındırma işlemi uygulamadan önce, yüzey üzerindeki doğal oksit tabakasını kontrol altına almak için 40s kadar amonyum peroksit uygulandı. Kristal $18 \mathrm{M} \Omega-$ $\mathrm{cm}$ özdirence sahip de-iyonize su ile yıkand1. Azot $\left(\mathrm{N}_{2}\right)$ gazı ile $3 \mathrm{dk}$ kurutma işleminden sonra kimyasal temizleme işlemi tamamlandı. Daha sonra, $10^{-6}$ Torr' luk yüksek vakum altında $\mathrm{p}$-Si mat yüzeyine, $\sim 99.999 \%$ saflıkta $\sim 1500 \AA$ kalınlığında Al kaplandı. $450^{\circ} \mathrm{C}$ de tavlanması ile Si yaprăğın üzerine çöktürülerek omik kontak elde edildi.

Elektro-eğirme yöntemi ile p-Si alttaş üzerine PVA' yı kaplama işlemi uygulandı. Kaplamadan önce pkatkılı Si alttaş, üzerindeki silisyum oksiti temizlemek için \%1 hidroflorik asit ile yıkand1. Daha sonra 0.1 g Çinko asetat, $0.9 \mathrm{~g}$ PVA ve $9 \mathrm{ml}$ deiyonize su ile karıştırıldı. Sonra $50^{\circ} \mathrm{C}$ de 2 saat karıştırıldıktan sonra PVA yoğun çözelti elde edildi. Peristaltik şırınga pompası kullanarak, öncü çözelti sabit akış hızı 0.02 $\mathrm{ml} / \mathrm{s}$ ' de $0.9 \mathrm{~mm}$ iç çaplı metal iğne $(10 \mathrm{ml})$ ile dağıtıldı. İğne, yüksek gerilim güç kaynağına bağlandı ve bir mengenede dikey yerleştirildi. Bir parça düz alüminyum yaprak, nanofiber parçacıklarını toplamak için iğnenin $18 \mathrm{~cm}$ altına yerleştirildi. p-katkılı Si alttaş, alüminyum yaprak üzerine yerleştirildi. $20 \mathrm{kV}$ yüksek voltajın üzerinde bir voltaj uygulayarak akışkan jet uçtan dışarı atıldı. Çözücü buharlaştı ve yüklü fiber, Si alttaş üzerine çökertildi.

p-Si alttaş üzerine 50 ve $150 \mathrm{~nm}$ kalınlıklarında Zn-katkılı PVA kaplandıktan sonra, aynı yüksek-vakum metal buharlaştırma sisteminde $10^{-6}$ Torr basınç altında bu tabaka üzerine $1 \mathrm{~mm}$ çapında ve $1500 \AA$ kalınlığında yüksek saflıktaki metal (Al) Schottky/doğrultucu kontak oluşturuldu. Sonuç olarak, $50 \mathrm{~nm}$ ve $150 \mathrm{~nm}$ kalınlıklarına sahip Al/(Zn-katkılı PVA)/p-Si MPS yapıları elde edildi. MPS yapıların frekansa bağlı kapasitans/kondüktans-voltaj ölçümlerini almak için HP 4192A LF Empedans Analizörü kullanıldı. 


\section{BULGULAR ve TARTIŞMA (RESULTS AND DISCUSSION)}

Al/PVA(Zn-katkıl1)/p-Si MPS yapıların ters ve doğru ön-gerilim altındaki C-G/ $\omega-\mathrm{V}$ ölçümleri $2 \mathrm{kHz}, 20$ $\mathrm{kHz}$ ve $200 \mathrm{kHz}$ frekansta, oda sicaklığında ve $-3.5 \mathrm{~V}$ ile $+5.5 \mathrm{~V}$ aralığında $0.05 \mathrm{~V}$ adımlarla gerçekleştirildi. Elde edilen bu deneysel C-G/ $\omega-\mathrm{V}$ karakteristikleri sırasıyla Şekil 1-2.(a-b)' de $\mathrm{MPS}_{1}$ ve $\mathrm{MPS}_{2}$ yap1 için verildi. Elde edilen eğrilerden kapasitans ve kondüktans değerlerinin önemli ölçüde frekansa bağlı olduğu görülmektedir. Şekil 1.(a-b)' de C-V eğrilerinin her birinde terslenim, tüketim ve yığılma bölgeleri açık olarak görülmektedir. Farklı frekanstaki $\mathrm{C}$ ve $\mathrm{G} / \omega$ değerlerinde meydana gelen değişmeler özellikle tüketim ve yığılma bölgelerinde olmaktadır. Tüketim bölgesinde $\mathrm{C}-\mathrm{V}$ eğrilerinde meydana gelen yarılma Si ile PVA arasında ve yasak enerji aralığında lokalize olmuş arayüzey durumlarının $\left(\mathrm{N}_{\mathrm{ss}}\right)$ varlı̆̆ına atfedilebilir [11].
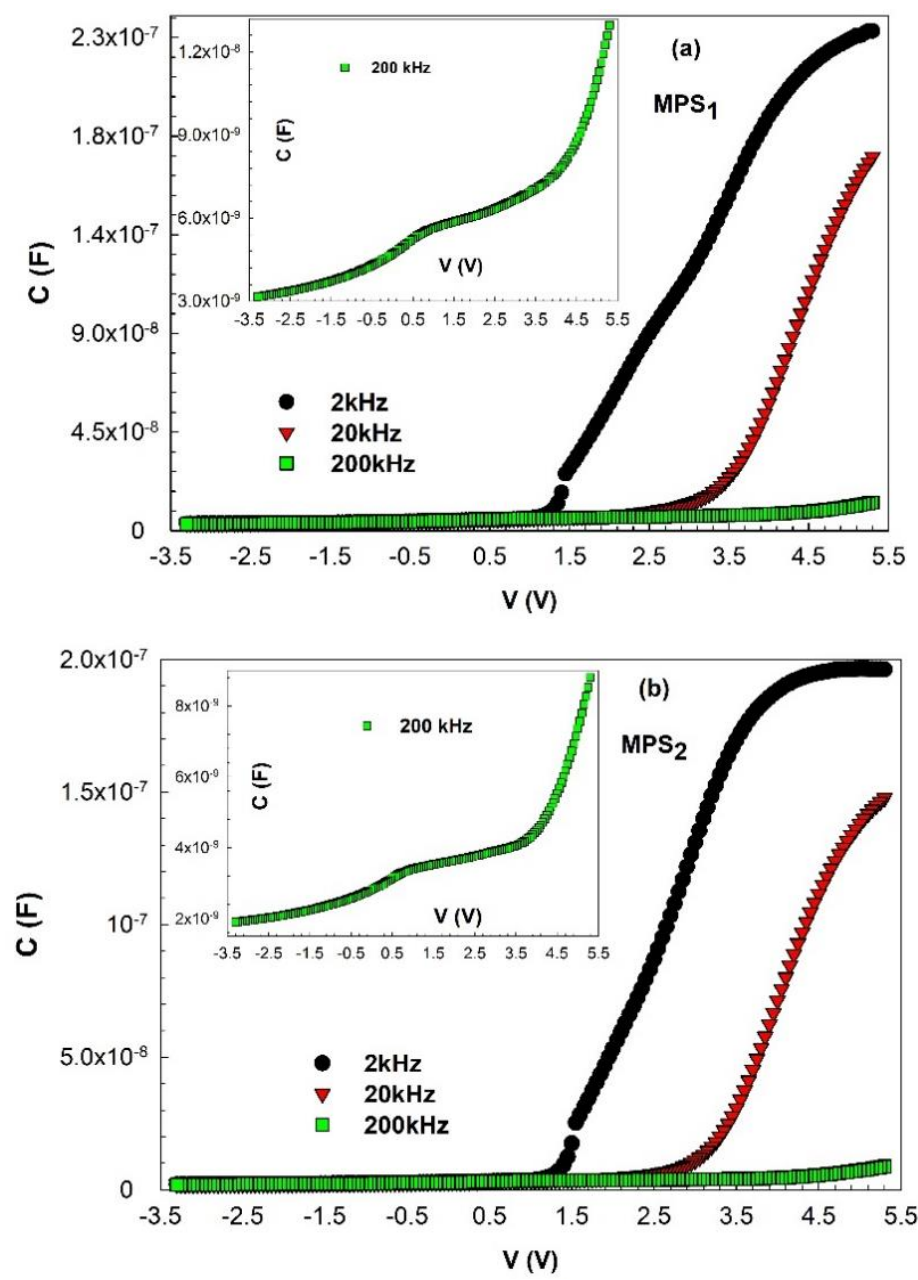

Şekil 1. Al/PVA (Zn-katk111)/p-Si (a) $M_{P S}$ ve (b) $M_{2} S_{2}$ için C-V eğrileri. 

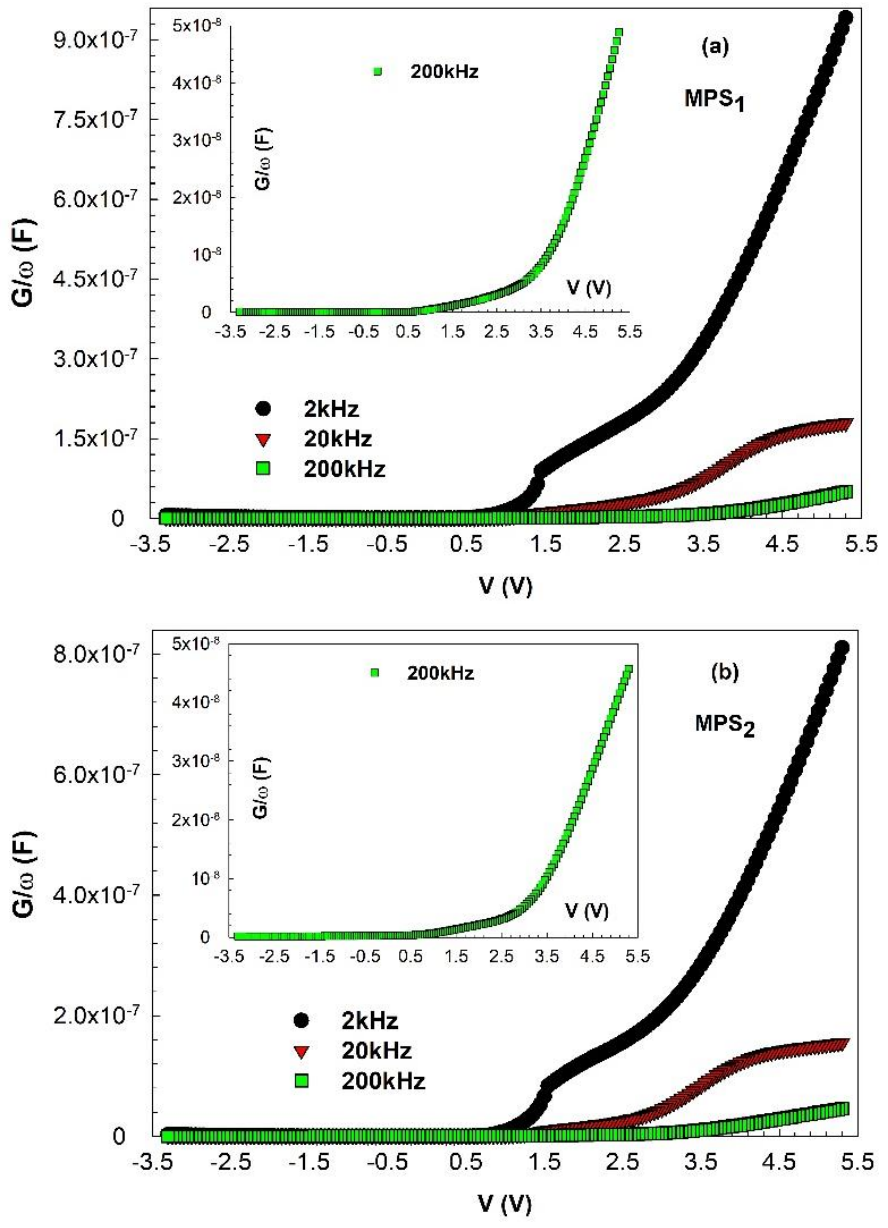

Şekil 2. A1/PVA (Zn-katk111)/p-Si (a) $\mathrm{MPS}_{1}$ ve (b) $\mathrm{MPS}_{2}$ için G/ $\omega$-V eğrileri.

Metal-yalıtkan/polimer-yariiletken yapılarda seri direnci hesaplamak için birçok yöntemi vardır [11]. Bu çalışmada, $\mathrm{R}_{\mathrm{i}}$ değerleri $\mathrm{C}-\mathrm{V}$ ölçümleri için Nicollian ve Brews tarafından geliştirilen ve diğer metotlara göre daha doğru, pratik, hızlı ve hassas olarak kabul gören admitans metodu kullanılarak $2 \mathrm{kHz}, 20 \mathrm{kHz}$ ve $200 \mathrm{kHz}$ frekansta voltaja bağlı olarak Eş.1 kullanılarak elde edildi [12].

$R_{i}=\frac{G_{m a}}{G_{m a}^{2}+\left(\omega C_{m a}\right)^{2}}$

Burada $C_{m a}$ ve $G_{m a}$ sırasıyla kuvvetli yığılma bölgesindeki kapasitans ve kondüktans değerleridir. $\mathrm{Bu}$ yapıların gerçek seri direnç değeri kuvvetli yı̆̆ılma bölgesindeki $C$ ve $\mathrm{G} / \omega$ değerlerinden elde edilir. Ancak bu formül voltaja bağlı $R_{i}$ değerlerini elde etmek içinde kullanılır ve bu yapının iletim mekanizmaları hakkında da bize önemli ipuçları verir. $\mathrm{R}_{\mathrm{i}}$ değerleri, hem frekansa hem de uygulanan gerilime oldukça bağlıdır. Her iki MPS yapısı için $\mathrm{R}_{\mathrm{i}}-\mathrm{V}$ grafiklerinde pikler gözlenmektedir. Frekansın artmasıyla yapıda görülen bu pik değerlerinin pozisyonlarının pozitif bölgeye kaydığı görülmektedir. Bu olay, Si/PVA arasında ve $\mathrm{E}_{\mathrm{g}}$ aralığında lokalize olmuş arayüzey durumlarının yeniden yapılanıp düzenlenmesine atfedilir. Ayrıca, voltaja bağlı $\mathrm{R}_{\mathrm{i}}{ }^{\prime}$ nin profili $\mathrm{N}_{\mathrm{ss}}$ ' in yasak enerji aralığındaki özel bir dağılımına atfedilebilir [13]. 

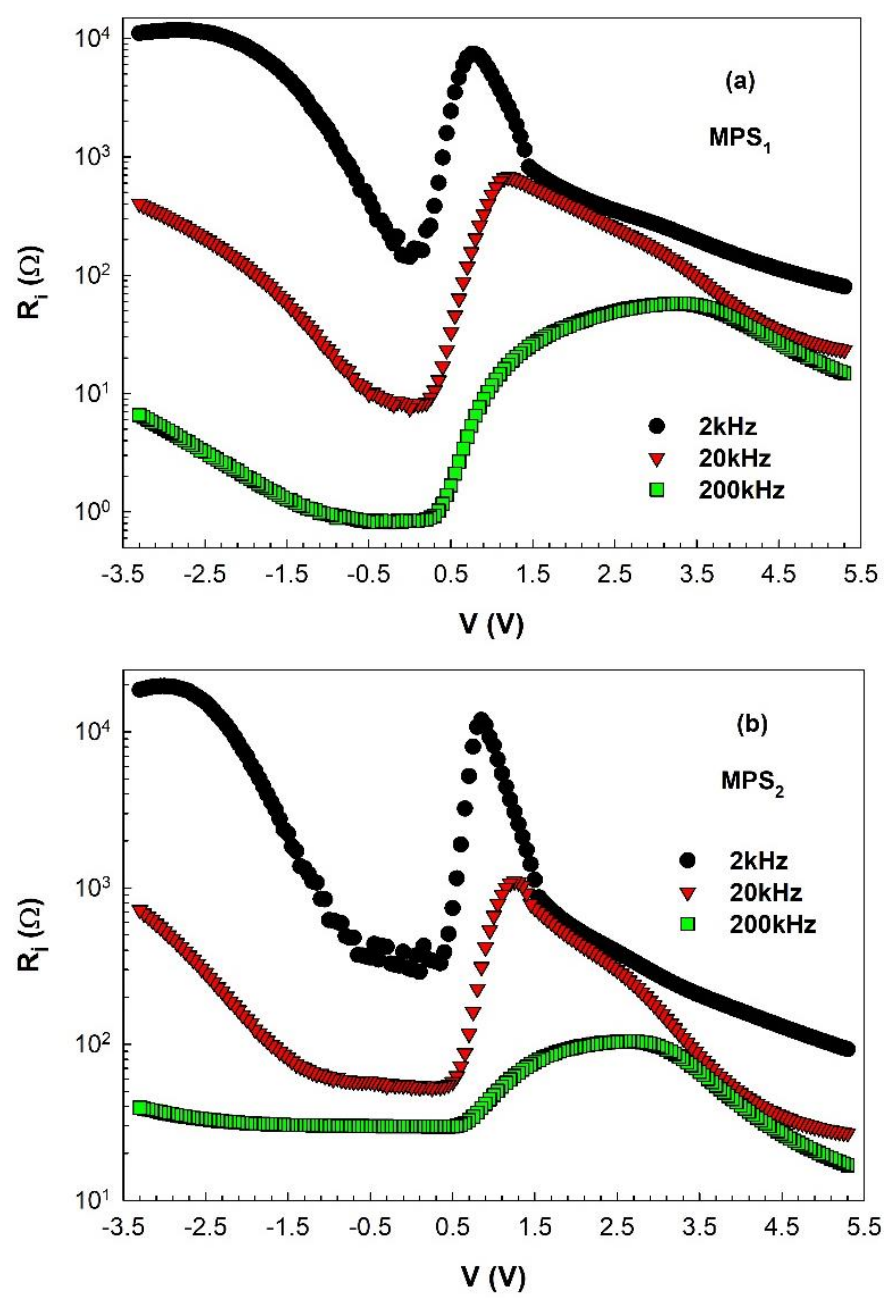

Şekil 3. Al/PVA (Zn-katk1lı)/p-Si (a) $M P S_{1}$ ve (b) $M P S_{2}$ için $\mathrm{R}_{\mathrm{i}}-\mathrm{V}$ eğrileri.

Düzeltilmiş admittans ifadesinin sanal ve gerçel kısmını $\left(\mathrm{Y}_{\mathrm{c}}=\mathrm{G}_{\mathrm{c}}+\mathrm{j} \omega \mathrm{C}_{\mathrm{c}}\right)$ elde etmek için ölçülen kapasitans $\left(\mathrm{C}_{\mathrm{m}}\right)$ ve kondüktans $\left(\mathrm{G}_{\mathrm{m}}\right)$ değerleri kullanılarak düzeltilmiş kapasitans $\left(\mathrm{C}_{\mathrm{c}}\right)$ ve iletkenlik $\left(\mathrm{G}_{\mathrm{c}}\right)$ değerleri sırasıyla Eş.2 ve Eş.3 kullanılarak $200 \mathrm{kHz}$ frekans için elde edilerek sırasıyla Şekil 4.a ve Şekil 4.b' de verildi [12].

$C_{C}=\frac{\left(G_{m}^{2}+\omega^{2} C_{m}^{2}\right) C_{m}}{a^{2}+\omega^{2} C_{m}^{2}}$

$G_{c}=\frac{\left(G_{m}^{2}+\omega^{2} C_{m}^{2}\right) a}{a^{2}+\omega^{2} C_{m}^{2}}$

Burada,

$a=G_{m}-\left(G_{m}^{2}+\omega^{2} C_{m}^{2}\right) R_{s}$

eşitliği ile verilen bir parametredir. Şekil 4.(a-b)' te görüldügü gibi her iki numune için düzeltilmiş kapasitans ve kondüktans değerleri özellikle yığılma bölgesinde artan voltaja bağlı olarak artmakta olduğu görülmektedir. Şekil 4.(a-b)' de $\mathrm{G}_{\mathrm{c}} / \omega-\mathrm{V}$ eğrilerinde tüketim bölgesinde pik gözlenmiştir. Bu pikler yasak enerji aralığındaki arayüzey durumlarının özel bir bölgede yoğunlaşmasından kaynaklanmaktadır. Şekil 4.(a-b)' de açıkça görüldüğü gibi $R_{s}$ etkisinin çok önemli olduğunu ve mutlaka bu $R_{s}$ değerinin etkisi dikkate alınarak ölçülen $\mathrm{C}_{\mathrm{m}}-\mathrm{G}_{\mathrm{m}} / \omega-\mathrm{V}$ eğrilerinin düzeltilmesini gerektiğini göstermektedir. $\mathrm{Bu}$ yapılmadığı takdirde elde edilen parametrelerin doğruluğu ve güvenilirliği tartışılır. 

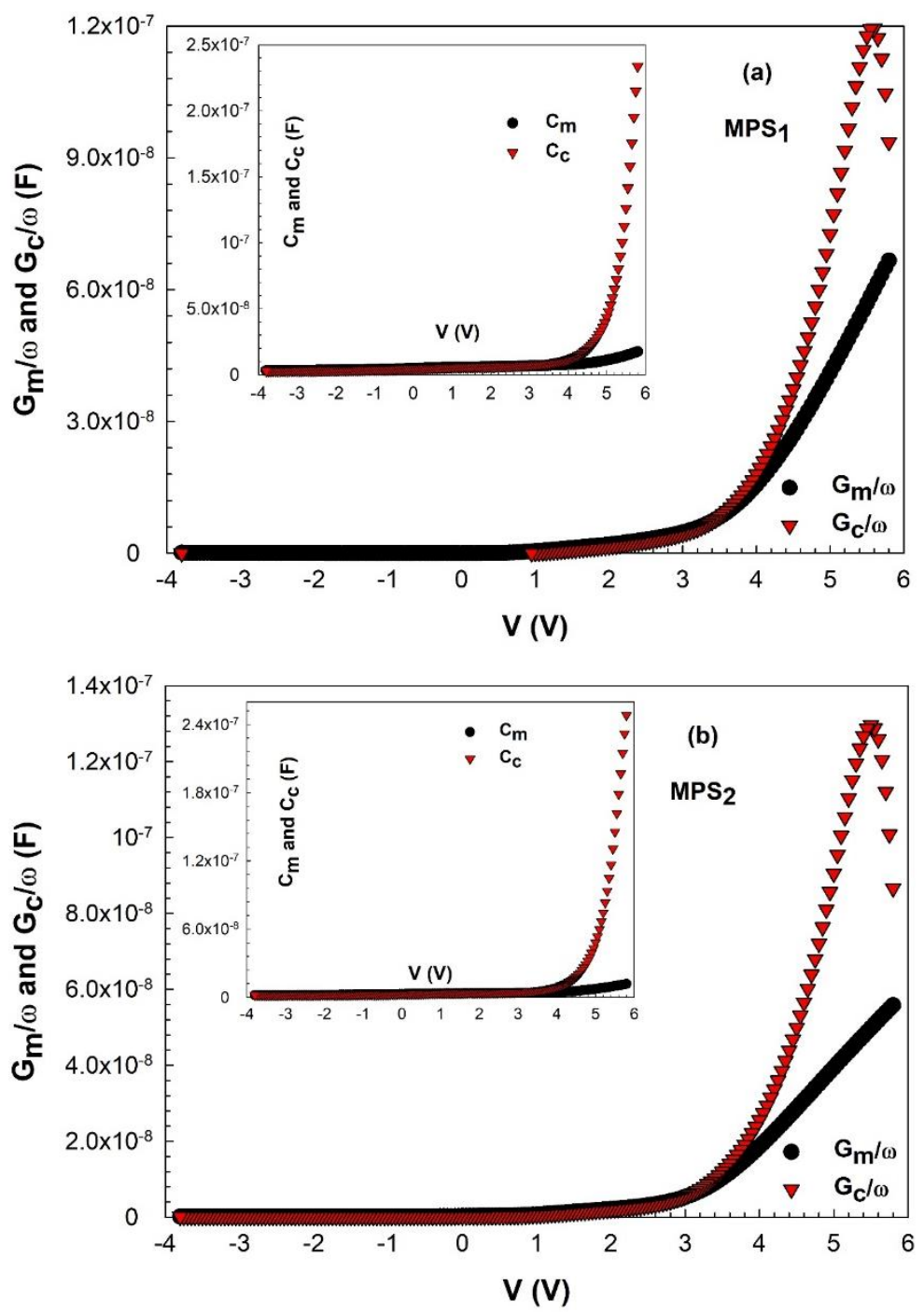

Şekil 4. Al/PVA (Zn-katk1lı)/p-Si (a) $M P S_{1}$ ve (b) $M_{P S}$ için $C_{m} / C_{c}-V$ ve $G_{m} / G_{c}-V$ eğrileri.

Hazırlanan MPS yapıların performansını seri direnç gibi arayüzey durumları oldukça etkilediği için bunların enerjiye veya voltaja bağlı dağ 1 lım profillerini elde etmek son derece önemlidir. Bu çalışmada, frekanstan dolayı gelen katkıyı elimine etmek için $\mathrm{C}-\mathrm{G} / \omega-\mathrm{V}$ ölçümleri $2 \mathrm{kHz}$ ve $200 \mathrm{kHz}$ frekansta gerçekleştirildi. $\mathrm{Bu}$ çalışmada arayüzey durumlarının dağılım profili düşük-yüksek frekans $\left(\mathrm{C}_{\mathrm{LF}}-\mathrm{C}_{\mathrm{HF}}\right)$ kapasitans metodu kullanılarak elde edildi. Bunların arasında $\mathrm{C}_{\mathrm{LF}}-\mathrm{C}_{\mathrm{HF}}$ kapasitans metodunun avantaj1, arayüzey tabakasının, yarıiletken alttaşın ve arayüzeyin birçok özelliğinin kolayca belirlenmesine izin vermesidir. Eş.5 kullanılarak arayüzey durumlarının voltaja bağlı dağılım profili elde edildi [12].

$N_{S S}=\frac{1}{q A}\left[\left(\frac{1}{C_{L F}}-\frac{1}{C_{o x}}\right)^{-1}-\left(\frac{1}{C_{H F}}-\frac{1}{C_{o x}}\right)^{-1}\right]$

Burada $C_{\mathrm{ox}}, \mathrm{C}_{\mathrm{LF}}$ ve $\mathrm{C}_{\mathrm{HF}}$ parametreleri sırasıyla arayüzey tabakanın kapasitansı, düşük ve yüksek frekans kapasitans değerleridir. Şekil 5' te görüldüğü gibi, MPS yapının kalınlığı artınca $\mathrm{N}_{\mathrm{ss}}$ değerleri artmaktadır. 

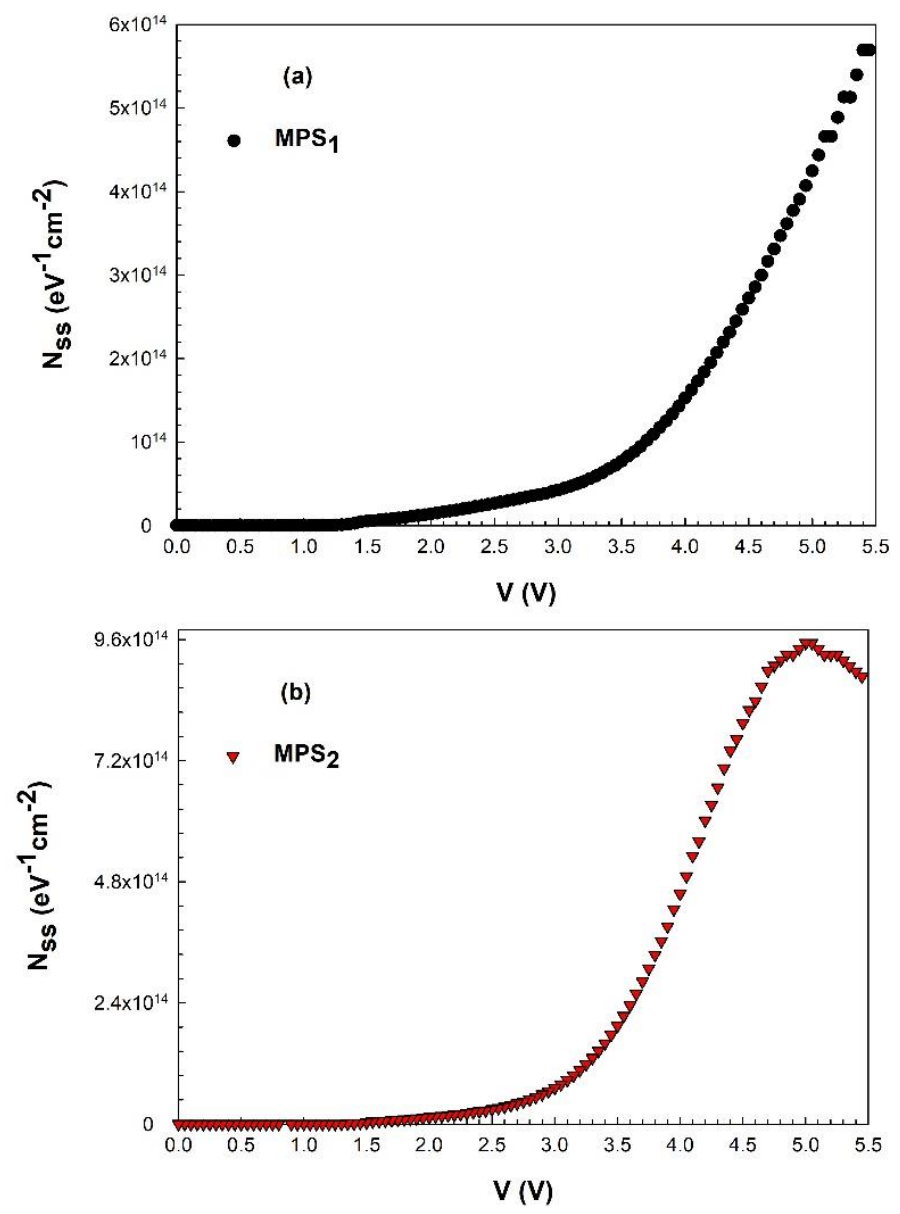

Şekil 5. Au/PVA (Zn-katkılı)/p-Si (a) $M_{P S}$ ve (b) $M_{P S}$ için $_{\mathrm{ss}^{-}}-\mathrm{V}$ grafiği.

$\mathrm{C}^{-2}-\mathrm{V}$ eğrileri $2 \mathrm{kHz}, 20 \mathrm{kHz}$ ve $200 \mathrm{kHz}$ frekans için iki MPS yapı içinde elde edildi. Şekil 6.(a-b)’ de görüldüğü gibi, $\mathrm{C}^{-2}-\mathrm{V}$ eğrileri her iki yapı içinde geniş bir voltaj aralığında lineer bir davranış göstermektedir. $\mathrm{Bu}$ lineer bölgede; MPS yapıları için $\mathrm{C}^{-2}$ ile $\mathrm{V}$ arasındaki ilişki Eş.6' daki gibi ifade edilebilir [13]:

$C^{-2}=\frac{2}{q \varepsilon_{S} \varepsilon_{o} A^{2} N_{A}}\left(V_{o}+V_{R}\right)$

Burada A doğrultucu kontak alanıdır, $\varepsilon_{\mathrm{s}}$ yariiletkenin dielektrik sabitidir. $\mathrm{N}_{\mathrm{A}}$ alıcı katkı atomlarının yoğunluğu, $V_{R}$ uygulanan ters ön-gerilimi ve $V_{o}$ ise $C^{-2}-V$ eğrisinin voltaj eksenine extrapole edilmesiyle elde edilen kurulma voltajıdır. $\mathrm{V}_{\mathrm{o}}$ ile $\mathrm{V}_{\mathrm{D}}$ difüzyon potansiyeli arasındaki ilişki,

$V_{o}=V_{D}-\frac{k T}{q}$

eşitliği ile verilir. Böylece farklı frekanslar için Eş.8 kullanılarak $\Phi_{B}$ değerleri elde edildi ve Çizelge 1' de verildi.

$\Phi_{B}=V_{D}+E_{F}-\Delta \Phi_{B}$ 
Burada $E_{F}$ yarriletkenin Fermi enerji seviyesidir ve $E_{F}=(k T / q) \ln \left(N_{v} / N_{A}\right)$ ile ifade edilir. $\Delta \Phi_{\mathrm{B}}$ hayali kuvveti engel düşmesi olup Eş.9’ dan elde edilebilir [13]:

$\Delta \Phi_{B}=\left(\frac{q E_{m}}{4 \pi \varepsilon_{s} \varepsilon_{o}}\right)^{1 / 2}$

Burada $E_{m}$ parametresi elektrik alanıdır ve Eş.10’ dan elde edilebilir.

$E_{m}=\left(\frac{2 q N_{A} V_{D}}{\varepsilon_{S} \varepsilon_{o}}\right)^{1 / 2}$

Farklı frekanslar için $\mathrm{C}^{-2}-\mathrm{V}$ eğrilerinden yapının elektriksel parametreleri elde edildi. Çizelge 1' den görüldüğü gibi, elde edilen bu değerler frekansa oldukça bağlilık göstermektedir. Son zamanlarda literatürde benzeri sonuçlar elde edilmiştir [14-20].

Çizelge1. $\mathrm{MPS}_{1}$ ve $\mathrm{MPS}_{2}$ için $\mathrm{C}^{-2}-\mathrm{V}$ eğrilerinden elde edilen çeşitli parametreler.

\begin{tabular}{|c|c|c|c|c|c|c|}
\hline \multicolumn{7}{|c|}{$\mathbf{M P S}_{1}$} \\
\hline $\begin{array}{l}\text { f } \\
(\mathrm{kHz})\end{array}$ & $\begin{array}{l}V_{D} \\
(e V)\end{array}$ & $\begin{array}{l}\mathrm{N}_{\mathrm{A}} \\
\left(\mathrm{cm}^{-3}\right)\end{array}$ & $\begin{array}{l}E_{F} \\
(e V)\end{array}$ & $\begin{array}{l}\mathbf{E}_{\mathrm{m}} \\
(\mathrm{V} / \mathrm{cm})\end{array}$ & $\begin{array}{l}\Phi_{B} \\
(e V)\end{array}$ & $\begin{array}{l}\mathrm{W}_{\mathrm{D}} \\
(\mathbf{c m})\end{array}$ \\
\hline 2 & 0.585 & $7.01 \times 10^{17}$ & 0.0940 & $3.53 \times 10^{5}$ & 0.614 & $3.31 \times 10^{-6}$ \\
\hline 20 & 0.631 & $6.99 \times 10^{17}$ & 0.0941 & $3.66 \times 10^{5}$ & 0.659 & $3.45 \times 10^{-6}$ \\
\hline 200 & 0.524 & $6.80 \times 10^{17}$ & 0.0950 & $3.29 \times 10^{5}$ & 0.556 & $3.18 \times 10^{-6}$ \\
\hline \multicolumn{7}{|c|}{$\mathbf{M P S}_{2}$} \\
\hline $\begin{array}{l}\mathbf{f} \\
(\mathbf{k H z})\end{array}$ & $\begin{array}{l}V_{D} \\
(e V)\end{array}$ & $\begin{array}{l}\mathrm{N}_{\mathrm{A}} \\
\left(\mathrm{cm}^{-3}\right)\end{array}$ & $\begin{array}{l}E_{F} \\
(e V)\end{array}$ & $\begin{array}{l}\mathbf{E}_{\mathrm{m}} \\
(\mathrm{V} / \mathrm{cm})\end{array}$ & $\begin{array}{l}\Phi_{B} \\
(e V)\end{array}$ & $\begin{array}{l}W_{D} \\
(\mathbf{c m})\end{array}$ \\
\hline 2 & 0.533 & $2.61 \times 10^{17}$ & 0.119 & $2.06 \times 10^{5}$ & 0.603 & $5.19 \times 10^{-6}$ \\
\hline 20 & 0.612 & $2.60 \times 10^{17}$ & 0.119 & $2.20 \times 10^{5}$ & 0.680 & $5.56 \times 10^{-6}$ \\
\hline 200 & 0.604 & $2.51 \times 10^{17}$ & 0.121 & $2.15 \times 10^{5}$ & 0.674 & $5.63 \times 10^{-6}$ \\
\hline
\end{tabular}



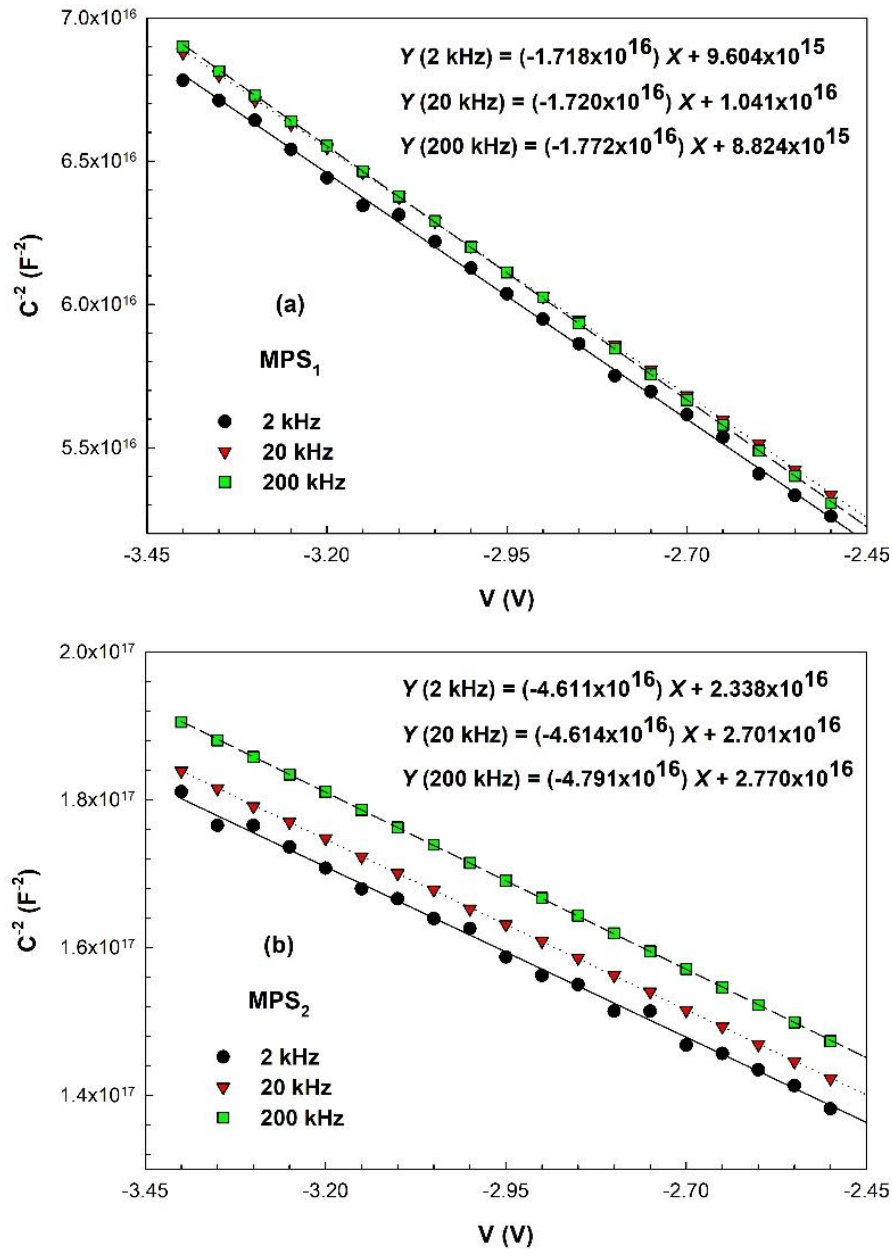

Şekil 6. Al/PVA (Zn-katkılı)/p-Si (a) $M_{P S}$ ve (b) $M_{2} S_{2}$ için $C^{-2}-V$ eğrileri.

\section{SONUÇ (CONCLUSION)}

$\mathrm{Bu}$ çalışmada, arayüzey polimer tabaka ve kalınlığının ölçülen $\mathrm{C}-\mathrm{G} / \omega-\mathrm{V}$ karakteristikleri üzerine etkisini araştırmak amacıyla 50 ve $150 \mathrm{~nm}$ kalınlıklarında (Zn-katkı1ı PVA) polimer ara yüzey tabakası Al/p-Si (MPS) arasına elektron-eğirme yöntemiyle büyütüldü. Bu iki yapının temel elektrik özellikleri, üç farklı frekansta $(2,20$ ve $200 \mathrm{kHz})$ ölçülen $\mathrm{C}-\mathrm{G} / \omega-\mathrm{V}$ ölçüm metodu kullanılarak geniş bir voltaj aralığında $(-3.5 /+5.5 \mathrm{~V})$ ve $50 \mathrm{mV}$ aralıklarla elde edilerek karşılaştıııldı. Temel yapı parametreleri olan $\mathrm{V}_{\mathrm{D}}, \mathrm{N}_{\mathrm{A}}, \mathrm{E}_{\mathrm{F}}$, $\mathrm{W}_{\mathrm{D}}$ ve $\Phi_{\mathrm{B}}$ değerleri terslenim bölgesi için elde edilen $\mathrm{C}^{-2}-\mathrm{V}$ grafiklerinin lineer kısımlarının kesim noktası ve eğimleri kullanılarak her frekans için elde edildi. Elde edilen bu değerlerin hem frekansa hem de arayüzey tabakasının kalınlığına oldukça bağlı olduğu gözlendi. Düşük frekanslarda elde edilen yüksek C ve $G$ değerleri arayüzey durumlarının varlığına ve onların yaşama ömürlerine atfedildi. $B u$ yüzden $R_{s}$ değerlerinin artan frekansla azalmaktadır. $\mathrm{C}_{\mathrm{LF}}-\mathrm{C}_{\mathrm{HF}}$ kapasitans metodu kullanılarak voltaja bağlı elde edilen $\mathrm{N}_{\mathrm{ss}}$ değerlerinin bölgeden bölgeye farklılık göstermesi ise onların polimer tabaka ile yariiletken arasında ve yasak enerji aralığına yerleşmiş arayüzey durumlarının özel bir dağılımına atfedildi. Elde edilen deneysel sonuçlar hem polimer arayüzey tabaka kalınlığının hem de $R_{s}$ değerlerinin $C-G / \omega-V$ ölçümleri üzerinde oldukça etkili olduğu ve bu yüzden elektriksel parametrelerin hesabında mutlaka dikkate alınması gerekmektedir.

\section{KAYNAKLAR (REFERENCES)}

[1] O. Çiçek, H. Uslu Tecimer, S.O. Tan, H. Tecimer, Ş. Altındal, İ. Uslu, Evaluation of electrical and photovoltaic behaviours as comparative of $\mathrm{Au} / \mathrm{n}-\mathrm{GaAs}$ (MS) diodes with and without pure and 
graphene (Gr)-doped polyvinyl alcohol (PVA) interfacial layer under dark and illuminated conditions. Composites Part B, 98 (2016) 260-268.

[2] O. Çiçek, H. Uslu Tecimer, S.O. Tan, H. Tecimer, İ. Orak, Ş. Altında, Synthesis and characterization of pure and graphene $(\mathrm{Gr})$-doped organic/polymer nanocomposites to investigate the electrical and photoconductivity properties of Au/n-GaAs structures. Composites Part B, 113 (2017) 14-23.

[3] H. Uslu Tecimer, M.A. Alper, H. Tecimer, S.O. Tan, Ş. Altındal, Integration of Zn-doped organic Polymer Nanocomposites Between Metal Semiconductor Structure to Reveal the Electrical Qualifications of The Diodes. Polymer Bulletin, https://doi.org/10.1007/s00289-018-2274-5 (2018).

[4] H. Tecimer, T. Tunç, Ş. Altındal, Investigation of photovoltaic effect on electric and dielectric properties of $\mathrm{Au} / \mathrm{n}$-Si Schottky barrier diodes with nickel (Ni)-zinc ( $\mathrm{Zn}$ ) doped organic interface layer. Journal of Materials Science: Materials in Electronics, 29 (2018) 3790-3799.

[5] H. Tecimer, S.O. Tan, Ş. Altındal, Frequency-Dependent Admittance Analysis of the MetalSemiconductor Structure With an Interlayer of Zn-Doped Organic Polymer Nanocomposites. IEEE Transactions on Electron Devices, 65 (2018) 231-236.

[6] S. Altındal Yerişkin, Arayüzey tabakalı metal/yarı iletken (MS) yapıların hazırlanması ve elektriksel ile dielektrik özelliklerinin geniş frekans ve voltaj aralığında incelenmesi. Gazi Üniversitesi Fen Bilimleri Enstitüsü Doktora Tezi (2017).

[7] H. G. Çetinkaya, Ş. Altındal, I. Orak, I. Uslu, Electrical characteristics of Au/n-Si (MS) Schottky Diodes (SDs) with and without different rates (graphene $+\mathrm{Ca}_{1.9} \mathrm{Pr}_{0.1} \mathrm{Co}_{4} \mathrm{O}_{\mathrm{x}}$ doped poly(vinyl alcohol)) interfacial layer. Journal of Materials Science: Materials in Electronics, 28 (2017) 7905-7911.

[8] E. E. Tanrıkulu, S. Demirezen, Ş. Altındal, İ. Uslu, On the anomalous peak and negative capacitance in the capacitance-voltage (C-V) plots of $\mathrm{Al} /(\% 7 \mathrm{Zn}-\mathrm{PVA}) / \mathrm{p}-\mathrm{Si}$ (MPS) structure. Journal of Materials Science: Materials in Electronics, 29 (2018) 2890-2898.

[9] İ. Taşçığlu, Ö. Tüzün Özmen, H.M. Şabanoğlu, E. Yağlığlu, Ş. Altındal, Frequency Dependent Electrical and Dielectric Properties of Au/P3HT:PCBM:F4-TCNQ/n-Si Schottky Barrier Diode. Journal of Electronic materials, 46 (2017) 2379-2386.

[10] S.A. Yerişkin, M. Balbaşı, İ. Orak, Frequency dependent electrical characteristics and origin of anomalous capacitance-voltage $(\mathrm{C}-\mathrm{V})$ peak in $\mathrm{Au} /($ graphene doped PVA)/n-Si capacitors. Journal of Materials Science: Materials in Electronics, 28 (2017) 7819-7826.

[11] H. Uslu, Au/(Co, Zn-Katkılı) Polivinil Alkol/n-Si Schottky Engel Diyotlarının Hazırlanması Ve Elektriksel Özelliklerinin Aydınlatma Şiddetine Bağlı İncelenmesi. Gazi Üniversitesi Fen Bilimleri Enstitüsü Doktora Tezi (2010).

[12] E.H. Nicollian, J.R. Brews, MOS Physics and Technology. John Wiley \& Sons, New York, 40-175, 222-226, 423-439 (1982).

[13] S.M. Sze, K.Ng Kwok, Physics of Semiconductor Devices. $3^{\text {rd }}$ ed.", John Wiley \& Sons, New Jersey, 362-390 (2007).

[14] M. Yildırım, The effect of interlayer thickness on frequency dependent electrical characteristics of $\mathrm{Al} / \mathrm{p}$-Si Schottky barrier diodes with $\mathrm{Bi}_{4} \mathrm{Ti}_{3} \mathrm{O}_{12}$ interlayer. Karaelmas Fen ve Mühendislik Dergisi, 6 (2016) 406-411.

[15] M. Sharma, S.K. Tripathi, Frequency and voltage dependence of admittance characteristics of $\mathrm{Al} / \mathrm{Al}_{2} \mathrm{O}_{3} / \mathrm{PVA} / \mathrm{n}-\mathrm{ZnSe}$ Schottky barrier diodes. Materials Science and Semiconductor Processing, 41 (2016) 155-161. 
[16] S. Demirezen, Au/(PrBaCoO nanofiber)/n-Si Yapılarda Seri Direnç ve Arayüzey Durumlarının Frekans ve Gerilime Bağlı Dağılım Profillerinin Farklı Metotlarla Elde Edilmesi. Gazi Üniversitesi Fen Bilimleri Dergisi: Part C, 5 (2017) 167-176.

[17] A. Tataroğlu, A. A. Al-Ghamdi, F. El-Tantawy, W. A. Farooq, F. Yakuphanoğlu, Analysis of interface states of $\mathrm{FeO}-\mathrm{Al}_{2} \mathrm{O}_{3}$ spinel composite film/p-Si diode by conductance technique. Applied Physics A, 122 (2016) 220(1-6).

[18] B. Kınacı, MS Yapısında Ara Yüzey Durumları ve Seri Dirence Bağlı Sicaklık ve Frekansın Elektriksel Karakterizasyonlar Üzerine Etkisi. Journal of Polytechnic, 20 (2) (2017) 313-318.

[19] Ç. Bilkan, Ş. Altındal. Investigation of the C-V characteristics that provides linearity in a large reverse bias region and the effects of series resistance, surface states and interlayer in $\mathrm{Au} / \mathrm{n}-\mathrm{Si} / \mathrm{Ag}$ diodes, Journal of Alloys and Compounds 708 (2017) 464-469.

[20] Ç. Bilkan, Ş. Altındal, Y.S. Azizian. Investigation of frequency and voltage dependence surface states and series resistance profiles using admittance measurements in $\mathrm{Al} / \mathrm{p}-\mathrm{Si}$ with $\mathrm{Co}_{3} \mathrm{O}_{4}-\mathrm{PVA}$ interlayer structures. Physica B, 515 (2017) 28-33. 